\title{
A quantitative investigation of additive noise reduction for active matrix flat-panel imagers using compensation lines
}

\author{
Y. El-Mohri, ${ }^{\text {a) }}$ L. E. Antonuk, Q. Zhao, M. Maolinbay, X. Rong, ${ }^{\text {b) }}$ K.-W. Jee, S. Nassif, \\ and C. Cionca \\ Department of Radiation Oncology, University of Michigan Medical Center, Ann Arbor, Michigan 48109
}

(Received 24 January 2000; accepted for publication 15 May 2000)

\begin{abstract}
A quantitative investigation of a technique for reducing correlated noise in indirect detection active matrix flat-panel imagers has been reported. Correlated noise in such systems arises from the coupling of electronic noise, originating from fluctuations in external sources such as power supplies and ambient electromagnetic sources, to the imaging array via its address lines. The noise reduction technique involves the use of signals from columns of compensation line pixels located in relatively close proximity to the columns of normal imaging pixels on the array. Compensation line pixels are designed to be as sensitive to externally-coupled noise as columns of normal imaging pixels but are insensitive to incident radiation. For each imaging pixel, correlated noise is removed by subtracting from the imaging pixel signal a signal derived from compensation line pixels located on the same row. The effectiveness of various implementations of this correction has been examined through measurements of signal and noise from individual pixels as well as of noise power spectra. These measurements were performed both in the absence of radiation as well as with $x$ rays. The effectiveness of the correction was also demonstrated qualitatively by means of an image of a hand phantom. It was found that the use of a single compensation line dramatically reduces external noise through removal of the correlated noise component. While this form of the correction increases non-radiation-related uncorrelated noise, the effect can be largely reduced through the introduction of multiple compensation lines. Finally, a position-dependent correction based on compensation lines on both sides of the array was found to be effective when the magnitude of the correlated noise varied linearly across the array. (c) 2000 American Association of Physicists in Medicine. [S0094-2405(00)01908-8]
\end{abstract}

Key words: active matrix flat-panel imager, amorphous silicon, compensation lines, correlated additive noise

\section{INTRODUCTION}

In recent years, the rapidly developing technology of active matrix flat-panel imagers (AMFPIs) has begun to undergo extensive evaluation for a variety of $\mathrm{x}$-ray imaging applications such as radiotherapy, ${ }^{1-5}$ radiography, ${ }^{6-10}$ fluoroscopy, ${ }^{6,7,9,10}$ and mammography. ${ }^{11,12}$ In the long term, the successful implementation of this technology for these and other applications will, at least partially, depend on the degree to which the quantitative performance of such imaging systems [as characterized, for example, by the detective quantum efficiency (DQE)] can be optimized. (The DQE can be defined as the ratio of the signal-to-noise at the output of the system to that at the input of the system, all squared.) Generally, it is highly desirable that the signal-to-noise ratio of an imaging system be as large as possible over a wide range of operational conditions. Towards achieving this goal, it is important that the imager be designed so as to provide a low level of additive noise. ${ }^{13}$ (Additive noise corresponds to the noise of the imaging system in the absence of radiation.) For example, for applications (such as fluoroscopy) involving low x-ray exposure per image, minimization of additive noise helps to insure that the performance of the system is limited by the noise associated with fluctuations in the inci- dent x-ray quanta, rather than by the additive noise- a desirable situation referred to as input-quantum-limited operation.

One particularly important component of the additive noise is the externally-coupled noise, $\sigma_{\text {ext }}{ }^{13}$ In an AMFPI system, an array of imaging pixels is read out by means of an electronic acquisition system. The Gate address line associated with each row of pixels is connected to external Gate driver circuitry and the Data address line associated with each column of pixels is connected to an external preamplifier circuit, one preamplifier per column. The $\sigma_{\text {ext }}$ noise component originates from sources (such as power supplies and ambient electromagnetic sources) which are external to the array and which couple to the preamplifiers via the Data lines. For noise that originates from power supplies, this coupling occurs at various sites in the array such as at the overlap of Gate and Data address lines and at the overlap of Gate and drain contacts in the thin-film transistor (TFT) switch typically present in each pixel. ${ }^{14,15}$ For noise that originates from ambient electromagnetic sources, coupling can occur through unshielded metal conductors present in the array or in the peripheral electronics. By virtue of how external noise originates, for readout of a given Gate line there is a high degree of correlation in the external noise appearing at different preamplifiers. Thus, the effect of external noise ap- 
pears largely as striations in an image and therefore affects image quality. The degree to which the striations are visible depends upon the relative magnitude of the correlated noise compared to the noise of the incident $\mathrm{x}$-ray quanta-thus making them more prominent at lower exposures such as those encountered in fluoroscopy. ${ }^{15,16}$ These considerations for $\sigma_{\text {ext }}$ apply equally to AMFPI designs which detect the radiation indirectly and directly.

Depending on the source of the externally-coupled noise, several methods are commonly employed to reduce the effect of this noise component on imaging performance. One method involves improving the quality of acquisition system power supplies, since voltage fluctuations in power supplies induce noise coupling through the Data line capacitance. Even a comparatively minor voltage fluctuation of $3 \mu \mathrm{V}$, when coupled to a typical Data line capacitance of $50 \mathrm{pF}$, yields a noise component of $\sim 950 e^{-}$. Another method involves providing electromagnetic shielding for sensitive electronic components of the imaging system. In particular, an array, with its metal grid structure of Gate and Data address lines, constitutes an antenna that couples external electromagnetic noise to the readout signal. For instance, a 200 $\mu \mathrm{m}$ pitch, $40 \times 40 \mathrm{~cm}^{2}$ array would have $\sim 1.6 \mathrm{~km}$ of metal lines. While peripheral electronics can be readily shielded, it is difficult to provide completely effective shielding for the array without serious degradation of the incident x-ray imaging information. Other methods of correlated noise reduction involve the use of correlated double sampling circuits implemented at the preamplifier level. ${ }^{17,18}$ Such circuits typically sample the signal from the Data line twice: once before and once during pixel charge readout. These two samples are then subtracted before digitization in order to cancel common noise components. While this technique can be effective for eliminating signal fluctuations whose temporal variation is slow compared to the two sampling intervals (i.e., lower frequency components), it does not remove higher frequency components of external noise.

A more robust method that offers the possibility of completely suppressing the correlated component of external noise at all frequencies involves using pixel signals from one or more Data lines as a basis for correcting pixel signals from other Data lines. This principle can be exploited through the incorporation of multiple columns of nonresponsive pixels (referred to as compensation lines) into the layout of an array. ${ }^{14,19,20}$ The design of these nonresponsive pixels is identical to the imaging pixels except for the fact that they are made to be insensitive to the incident radiation and thus produce no x-ray signal. Figure 1 illustrates an example of an indirect detection array with a single compensation line situated at the periphery of the array. As in the case of the dual-correlated sampling technique, for a given row of imaging pixels the signal from a compensation line pixel can be subtracted (digitally or prior to digitization) from that of each imaging pixel along the row. While this subtraction eliminates the correlated noise component, it increases the uncorrelated noise. Fortunately, this increase can be greatly suppressed through the incorporation of multiple compensation lines at the periphery of the array. For a given row of imag-

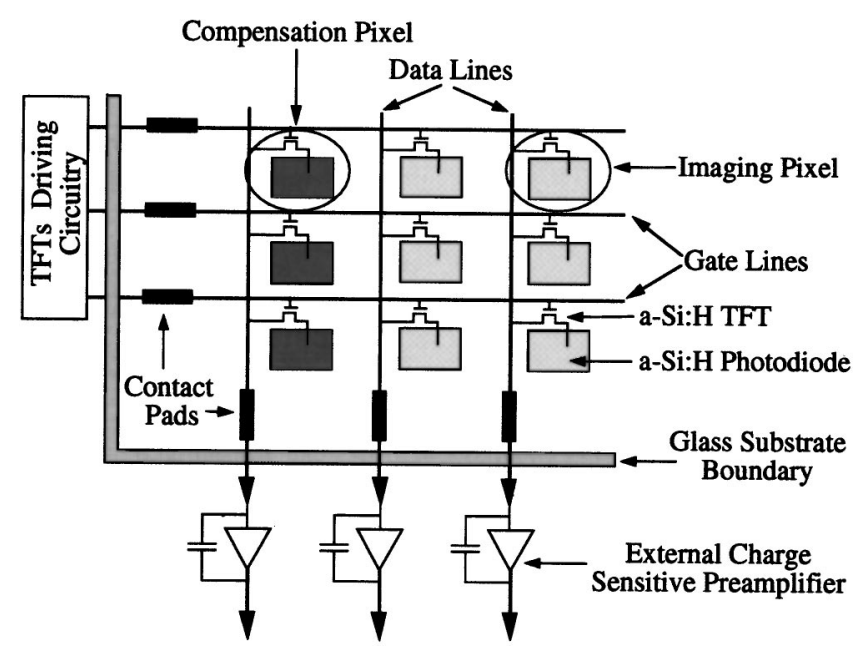

FIG. 1. Schematic diagram of a corner of an indirect detection, active matrix flat-panel array containing a single compensation line-a column of pixels which are similar in design to normal array pixels, but which are nonresponsive to the incident radiation. Electronic circuits peripheral to the array are also shown in the figure.

ing pixels, a correction based on the use of an average signal determined using all of the corresponding compensation line pixels for that row results in a net reduction of the uncorrelated noise contribution.

In this article, a study of the effectiveness of employing compensation lines to suppress external noise for indirect detection, active matrix flat-panel imagers is reported. In this study, the suppression of external noise is quantified through measurements of pixel signal, of the noise in the signal from individual pixels (which, for the purposes of this article, is referred to as "pixel noise"), as well as noise power spectra (NPS). These measurements were performed both in the absence of radiation (where system noise consists solely of additive noise) and with $\mathrm{x}$ rays (where system noise includes additive noise and x-ray quantum noise). In addition, the effectiveness of noise suppression using compensation lines is demonstrated qualitatively by means of an image obtained with an anthropomorphic phantom.

\section{METHODS AND MATERIALS}

\section{A. Description of the imaging system}

The indirect detection active matrix flat-panel imager employed in the present study consists of four main components: a pixelated array which incorporates amorphous silicon $(a-\mathrm{Si}: \mathrm{H})$ TFTs and photodiodes deposited on a glass substrate; a Lanex Regular phosphor screen (Eastman Kodak; $\sim 70 \mathrm{mg} / \mathrm{cm}^{2} \mathrm{Gd}_{2} \mathrm{O}_{2} \mathrm{~S}: \mathrm{Tb}$ ), which is placed in contact with the array surface and which serves to convert $\mathrm{x}$ rays to optical photons; an electronic acquisition system which controls the operation of the array and extracts and digitizes pixel charge $;^{21}$ and a host computer which controls the acquisition electronics and processes digital pixel data.

The array, which was originally developed for radiotherapy imaging, has an active area of $26.0 \times 26.0 \mathrm{~cm}^{2}$, and consists of a matrix of $512 \times 512$ pixels with a pixel pitch of 
TABLE I. Design specifications of the array employed in the flat-panel imager used in the measurements. The pixel charge capacity corresponds to a photodiode reverse bias voltage of $-5 \mathrm{~V}$ (Ref. 1). The maximum frame rate is based on a TFT-on voltage of $10 \mathrm{~V}$, and a pixel sampling time of five time constants (Ref. 6). The pixel fill factor is defined as the ratio of the optically sensitive area of the pixel to the total pixel area.

\begin{tabular}{lc}
\hline \hline Pixel format $($ Data $\times$ Gate $)$ & $512 \times 512$ \\
Pixel pitch & $508 \mu \mathrm{m}$ \\
Array dimensions & $26.0 \times 26.0 \mathrm{~cm}^{2}$ \\
Photodiode geometric area & $\sim 0.22 \mathrm{~mm}^{2}$ \\
Fill factor & $\sim 0.84$ \\
Nominal photodiode capacitance & $\sim 16.9 \mathrm{pF}$ \\
Pixel charge capacity $\left(V_{\text {bias }}=-5 \mathrm{~V}\right)$ & $\sim 90 \mathrm{pC}$ \\
Pixel dark current $\left(V_{\text {bias }}=-5 \mathrm{~V}\right)$ & $\sim 0.3 \mathrm{pA} / \mathrm{mm}^{2}$ \\
Maximum frame rate & $\sim 22 \mathrm{fps}$ \\
\hline \hline
\end{tabular}

$508 \mu \mathrm{m}$. Detailed specifications of the array are given in Table I. Each pixel consists of a light sensitive photodiode coupled to a thin-film transistor. While the photodiode serves both to sense radiation as well as to store charge, the TFT acts as a switch enabling the readout of the accumulated charge on a row-by-row basis under the control of the acquisition system. A reverse-bias voltage applied to the photodiodes results in the collection of charge created by the incident radiation. Array readout is typically performed for one row of pixels at a time by integrating the charge from each pixel in the charge sensitive preamplifier circuit located at the end of the corresponding Data line. Analog signals from the preamplifiers are multiplexed and digitized to an effective 15 bit-resolution. Addressing all, or some fraction, of the rows on the array constitutes a "readout cycle" and the corresponding information, if saved, is termed a "data frame."

\section{B. Experimental setup}

$\mathrm{X}$ ray measurements were performed with an $\mathrm{x}$-ray tube (Dunlee PX1415) which was powered by a high frequency generator (Picker MTX380) and which was suspended above the array at a distance of $\sim 116 \mathrm{~cm}$. Synchronization between the operation of the $\mathrm{x}$-ray source and the readout of the array was orchestrated by the acquisition software. ${ }^{6}$ For the pixel signal, pixel noise, and NPS measurements with $\mathrm{x}$ rays, an $18 \mathrm{~cm}$ thick tissue-equivalent solid water phantom (Gammex R.M.I., 457) was positioned $\sim 16 \mathrm{~cm}$ above the imaging array in order to lower the exposure. Throughout these measurements, this source, which was operated in radiographic mode, was used to provide a fixed amount of radiation per image frame $-\sim 1.4 \mathrm{mAs}$ at $70 \mathrm{kVp}$ corresponding to an $\sim 80 \mu \mathrm{R}$ exposure to the detector.

For a given set of experimental conditions, if more than a single data frame was acquired, the data frames were obtained in a single acquisition sequence. For a given sequence, a large number of readout cycles were performed in the absence of radiation prior to the acquisition of the first data frame in order to remove trapped charge as well as to establish equilibrium between charge trapping and charge release in the photodiodes. ${ }^{6}$ The number of initializing cycles performed was 200 (when 256 or 512 Gate line were read out per cycle) or 2000 (when 20 Gate lines were read out per cycle). Within an acquisition sequence, an additional 20 readout cycles were performed prior to each subsequent data frame in order to reestablish equilibrium between charge trapping and release.

The time per readout cycle (frame time) varied depending on the number of Gate lines addressed and included a delay of $80 \mathrm{~ms}$ to accommodate the duration of the radiographic $\mathrm{x}$-ray exposure. For all measurements, the TFT-on and TFToff voltages applied to the Gate lines were +10 and $-8 \mathrm{~V}$, respectively. ${ }^{6}$ The pixel photodiode reverse bias voltage was $-5 \mathrm{~V}$ corresponding to a total pixel charge capacity of $\sim 90$ pC (Ref. 1). The charge capacity of the preamplifier circuit $(\sim 5 \mathrm{pC})$ resulted in a conversion factor of $\sim 1125 e^{-}$per ADC channel. (The system was operated so as to insure that the preamplifiers never saturated.) The integration time of the preamplifier circuit (300 $\mu \mathrm{s})$ was chosen to insure complete collection of the pixel imaging charge.

In order to enhance the correlated noise contribution to external noise and therefore test the efficiency of the compensation line method in suppressing correlated noise, an external noise source was added providing a sinusoidal timedependent signal of $60 \mathrm{~Hz}$ frequency. (This was done for every measurement except those associated with the NPS analysis.) This artificially induced noise coupled to the preamplifiers each time a row of pixels was read out. Since the time interval between the readout of consecutive rows is $\sim 2.25 \mathrm{~ms}$, which corresponds to a higher frequency $(\sim 440$ $\mathrm{Hz})$ than that of the noise source $(60 \mathrm{~Hz})$, signal variations induced by the external source are adequately sampled by the acquisition system.

\section{Compensation line corrections}

In the present study, the compensation lines used were columns of normal pixels situated on the left and right sides of the array. Up to 14 Data lines on each side were treated as compensation lines. The compensation lines were kept in the dark, outside of the radiation field, and far away from the phosphor screen used in the experiments. (For all x-ray measurements, the screen covered a region of the array corresponding to Data line addresses 109 to 391). With this arrangement, it was empirically verified that the signal from the compensation line pixels contained negligible contributions from ambient light or $\mathrm{x}$-ray radiation.

A number of techniques for employing the compensation line information to correct for correlated noise were examined. One correction technique consisted of subtracting the signal from a single compensation line. In a second technique, the signal used for subtraction was the average signal from a number of compensation lines located on one side of the array. A third technique was based upon the premise that the magnitude of the correlated noise varied in a linear manner along the length of the Gate lines. This method involved the use of the pair of averaged signals derived from the compensation lines on the two sides of the array, where the contribution from each side depended upon its proximity to the imaging pixel. For a given pixel along a Gate line, the applied correction to the corresponding signal is 


$$
S_{\mathrm{corr}}=S_{i}-\left(\frac{x_{2}-i}{x_{2}-x_{1}} C_{1}+\frac{i-x_{1}}{x_{2}-x_{1}} C_{2}\right),
$$

where $S_{i}$ is imaging pixel signal at position $i$ along the Gate line, $x_{1}$ and $x_{2}$ are the central positions of the groups of compensation line pixels on either side of the array, respectively, and $C_{1}$ and $C_{2}$ are the averaged correction signals from the two groups of compensation line pixels, respectively. All positions are in units of Data line addresses.

The effectiveness of these various techniques on reducing the correlated noise component was examined by comparing pixel signal, pixel noise, NPS, and image results obtained with and without the application of compensation line corrections. For those results obtained with compensation line corrections, the correction was always performed to the raw pixel signal data prior to any other processing of that data. For example, image processing, consisting of the application of offset and gain corrections to the data, ${ }^{22}$ was performed for the pixel signal, NPS, and image comparisons. The image processing offset and gain calibration constants used when compensation line corrections were applied were determined using the same form of compensation correction whereas the calibration constants used when compensation corrections were not applied were determined without any correction. For the NPS analysis, when compensation line corrections were applied, the image processing steps were performed after these corrections and before the steps associated with this particular analysis.

\section{Pixel signal measurements}

An examination of the effect of compensation line corrections on pixel signal measurements was performed in order to provide a quantitative demonstration of the application and efficiency of such corrections both in the absence of radiation (dark field) as well as with x rays (flood field). For each condition, a single frame was acquired to provide pixel signal data. Each data frame consisted of a block of pixels corresponding to a region of 512 Data lines by 256 Gate lines obtained at a frame time of $\sim 0.65 \mathrm{~s}$. For the flood field data frame, offset and gain corrections for each pixel were applied, while for the dark field data frame only the offset corrections were applied. These offsets and gains correct for stationary variations in pixel response, for channel-tochannel variations among the preamplifier electronics, and for structure noise inherent to the phosphor screen. The offset and gain corrections were themselves derived from the average of 50 dark and 50 flood field data frames, respectively, which were obtained immediately prior to the acquisition of the pixel signal data. The 50 flood field frames used to derive the gain corrections were obtained at the same exposure level as the corresponding pixel signal measurements. Data from a single Data line (256 pixels) was extracted from each of the pixel signal frames and analyzed in order to illustrate correlated noise contributions to pixel signal. The compensation correction employed in these measurements involved the use of a single compensation line.

\section{E. Pixel noise measurements}

For the three compensation line correction techniques, the effectiveness of each technique was quantified through measurements of individual pixel noise performed in the absence of radiation as well as with $\mathrm{x}$ rays. For each condition, 200 data frames were obtained. Each data frame corresponded to a region of 512 Data lines by 20 Gate lines and was obtained at a frame time of $\sim 0.12 \mathrm{~s}$. For every pixel, signals derived from the data frames were used to determine a measure of pixel noise. For results which include a compensation line correction, the correction was applied to each data frame prior to the determination of pixel noise. The noise analysis consisted of organizing the data frames into ten sets of 20 consecutive data frames. This division was performed in order to minimize the contribution of pixel signal drift to pixel noise, given that several minutes were typically required to obtain the data. For each set of data frames, the standard deviation in the mean signal of a pixel was calculated for a selected number of correctly functioning pixels. Finally, for each pixel the results from all sets were then averaged to yield a value of noise.

\section{F. NPS measurements}

The measurement of NPS involved the acquisition of 50 data frames, $512 \times 256$ pixels (Data $\times$ Gate) per frame, in the absence of radiation as well as with $\mathrm{x}$ rays at a frame time of $\sim 0.65 \mathrm{~s}$. For each frame of this NPS data, the analysis was performed to a central region of the array consisting of 256 $\times 256$ pixels. For all of these measurements, the aforementioned external noise source was not used since the imaging system already exhibited a $60 \mathrm{~Hz}$ noise correlation that was easily quantified by the NPS analysis. Offset and gain corrections were obtained and applied to the data in the manner previously described for the pixel signal measurements. After application of offset and gain corrections, pixel signal values were converted to units of electrons by means of the measured calibration of the preamplifiers. A $3 \times 3$ median filter was applied to a small number of pixel columns corresponding to noisy preamplifier channels. Finally, individual pixel defects were identified, and a $3 \times 3$ median filter was applied to these pixels, affecting less than $0.1 \%$ of the total NPS data.

After the application of offset and gain corrections and the filters, NPS were determined using a synthesized slit technique. ${ }^{23-25}$ This involved the selection of 400 nonoverlapping slits, each of dimension $32 \times 256$ pixels $(L \times n)$, from the data frames (eight slits per data frame). Each slit was then summed along the $L$ direction to form an $n$-point realization. To remove low frequency background trends, a linear fit was performed to the realizations (of width $n$ =256) and subtracted to yield zero-mean, detrended data. A Hanning window function was then applied to remove distortions in the estimated spectra due to the finite-length realizations. The 400 realizations were Fourier transformed and normalized to yield an ensemble of 400 power spectra, which were then averaged to yield the measured NPS. These resulting NPS consisted of 128 points at a frequency interval 
of $0.0077 \mathrm{~mm}^{-1}$ up to the Nyquist frequency $\left(\sim 1 \mathrm{~mm}^{-1}\right)$. The analysis was performed for realizations running along the Data line direction in order to observe the effect of external noise correlation. For purposes of comparison, NPS analysis was also performed for realizations running along the orthogonal (Gate line) direction.

In the case of this analysis, the compensation line correction technique used corresponded to that of Eq. (1). This involved the use of 14 compensation lines located on each side of the region from which the NPS data was obtained.

\section{G. Imaging example}

In order to demonstrate the effectiveness of compensation line corrections, a hand phantom was imaged in presence of
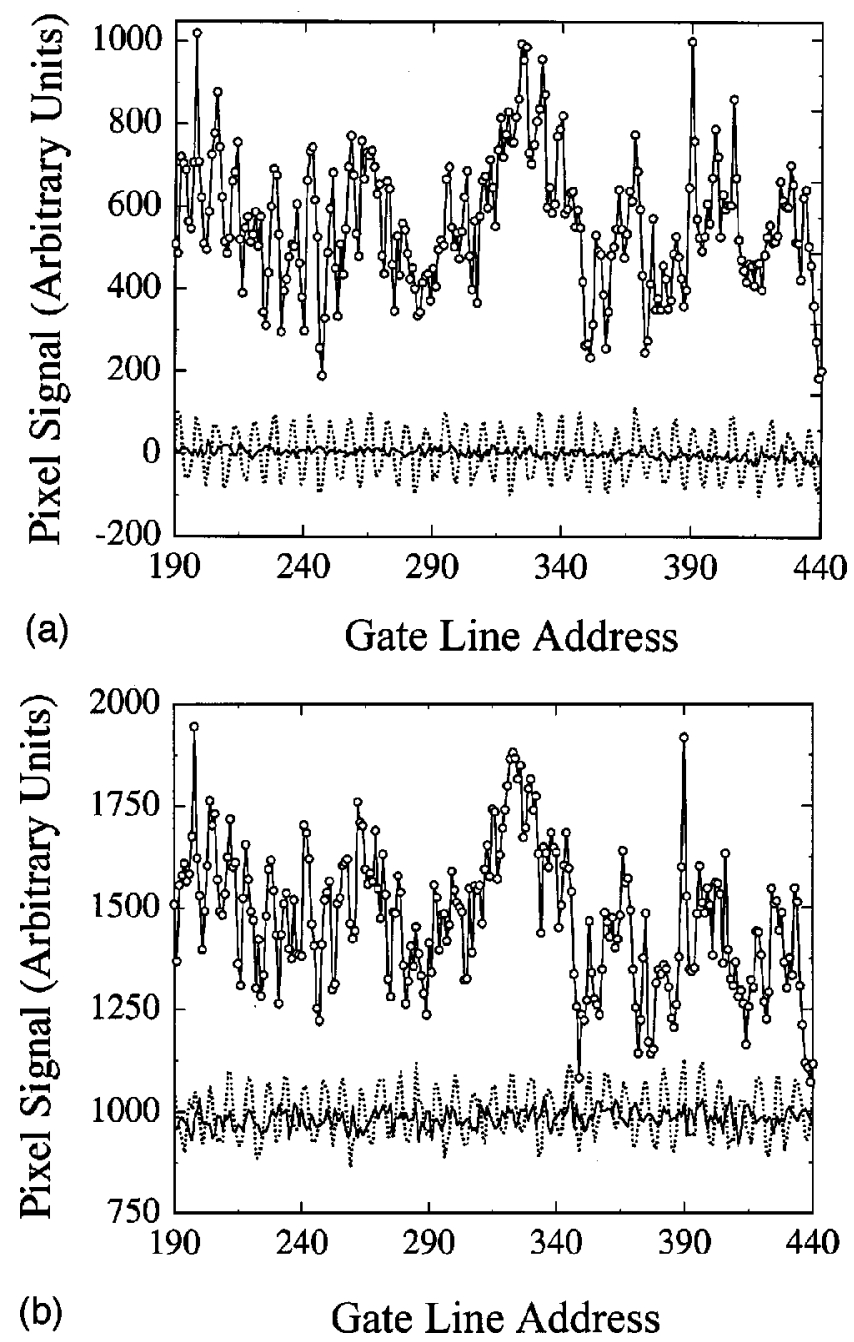

FIG. 2. Signal (in arbitrary units) from individual pixels along a single Data line plotted as a function of their corresponding Gate line address. Results are shown corresponding to data acquired (a) in the absence of radiation and (b) with $\mathrm{x}$ rays. In each graph, the circles correspond to pixel data in the absence of any correction (i.e., prior to the application of compensation line corrections or offset and gain corrections). The dashed curves correspond to the application of offset corrections (for both dark and flood field data) and gain corrections (for flood field data only) while the solid curves correspond to the application of gain and offset corrections as well as of a compensation line correction. Note that the vertical scale for both graphs corresponds to the same signal range. the external noise source. The image was taken at $70 \mathrm{kVp}$ using a $0.5 \mathrm{mAs}$ exposure. An additional aluminum filter $(\sim 5.5 \mathrm{~mm}$ thick) located near the source was used to reduce the exposure so as to accentuate the visibility of the correlated noise. All $512 \times 512$ pixels of the array were read out at a frame time of $\sim 1.2 \mathrm{~s}$. Prior to image acquisition, 50 dark and 50 flood fields were acquired to obtain offset and gain calibration constants that were applied to the raw image data. The compensation line correction technique used corresponded to that employed in the NPS analysis.

\section{RESULTS}

\section{A. Pixel signal measurements}

In Fig. 2(a), measured dark field signal for consecutive pixels along a single representative Data line is plotted as a function of Gate line address. In this figure, the data indicated by circles corresponds to pixel signals in the absence of either compensation line corrections or offset corrections (i.e., raw signal data). Application of the offset correction to the raw data removes the pixel-to-pixel variations due to leakage currents leaving a periodic component, illustrated by the dashed line in Fig. 2(a), that is largely due to noise pickup from the external noise source $(60 \mathrm{~Hz})$. These oscillations are largely suppressed (as indicated by the continuous line in the figure) when a compensation line correction (based on the signals from a single compensation line) is applied prior to the offset correction. Similarly, as illustrated in Fig. 2(b), the application of offset and gain corrections to raw flood field pixel data (circles) results in pixel data (dashed line) exhibiting large noise contributions from the external source. However, when both a compensation line correction and offset and gain corrections are applied to the

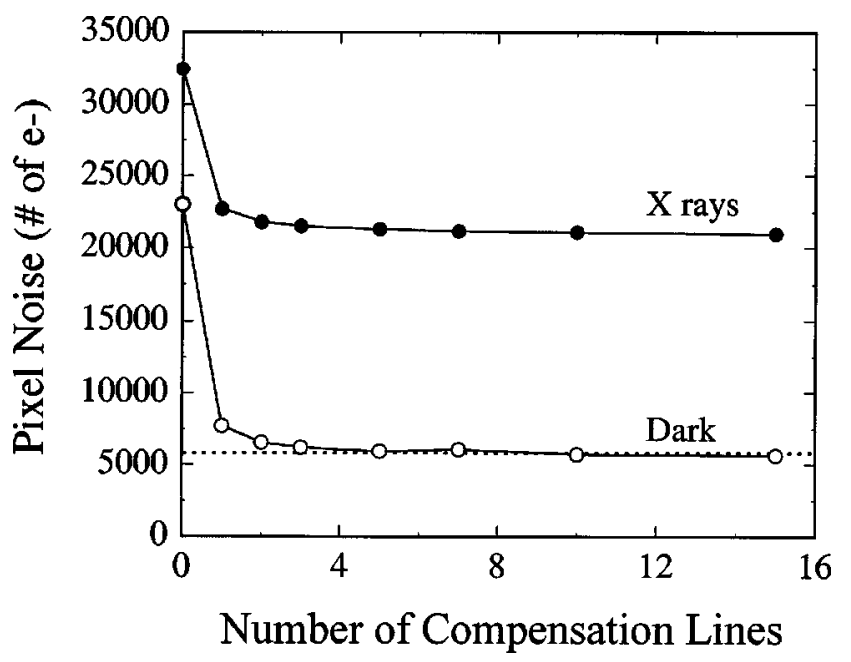

FIG. 3. Noise from an individual pixel plotted as a function of the number of compensation lines used in the compensation line correction. (The results shown at a value of " 0 ", lines correspond to the absence of this correction.) Results, obtained in the presence of an additional external noise source, are shown corresponding to measurements taken with no radiation (open circles) as well as with $\mathrm{x}$ rays (solid circles). For purposes of comparison, the value of the pixel noise in the absence of radiation, with no external noise source and with no compensation line correction, is indicated by the dashed horizontal line. 
raw flood field pixel data, significant reduction of pixel-topixel fluctuations is observed (solid line), leaving only small variations in the data. These variations most likely originate largely from random fluctuations in the x-ray quanta.

\section{B. Pixel noise measurements}

Figure 3 shows measured pixel noise in the absence of radiation as well as with $\mathrm{x}$ rays from an individual imaging pixel whose behavior was representative of correctly functioning pixels on the array. The results shown were obtained both with and without the use of a compensation line correction and are plotted as a function of the number of Data lines used in the correction. The data points appearing at a value of " 0 " along the horizontal axis correspond to the absence of the correction. For the other results corresponding to the inclusion of the correction, the Data lines used as compensation lines were taken from a single side of the array. For both the dark and x-ray results, the use of a single compensation line dramatically reduces the system noise by removing correlated external noise contributions. However, the subtraction of the compensation line signal from the imaging pixel signal increases non-radiation-related uncorrelated noise contributions by $\sqrt{2}$. Fortunately, the introduction of additional compensation lines gradually reduces this effect such that, by $\sim 5$ compensation lines, the measured noise has been reduced at least down to the level achievable in the absence of the external $60 \mathrm{~Hz}$ noise source. This is explicitly demonstrated in the case of the dark noise measurements where the data points eventually reach a noise level that is lower than the pixel noise observed in absence of the external source and without the compensation line correction (indicated by the dashed horizontal line in the figure).

Figure 4 shows the measured noise of pixels along a single Gate line, obtained in the absence of radiation as well as with $\mathrm{x}$ rays, as a function of Data line address. For the dark measurements with the external noise source present, the level of pixel noise in the absence of any compensation line correction (solid circles) is observed to be considerably reduced through the application of a correction based on Eq. (1) (open circles). These reduced noise values are comparable to (or slightly lower than) the noise observed in the absence of the external source and without the compensation line correction (crosses). A similar trend of significant noise reduction is observed for the $\mathrm{x}$-ray results where pixel noise before compensation line corrections (solid diamonds) is reduced to a level dominated by quantum noise (open diamonds) when corrections are applied. It is of interest to point out that a slight left-to-right asymmetry in the uncorrected dark noise results (arising from a nonuniform spatial contribution from the external noise source) is removed when the compensation line correction based on Eq. (1) is applied, as illustrated in Fig. 4. However, this is not the case when a correction based on compensation lines from only one side of the array is applied (not shown).
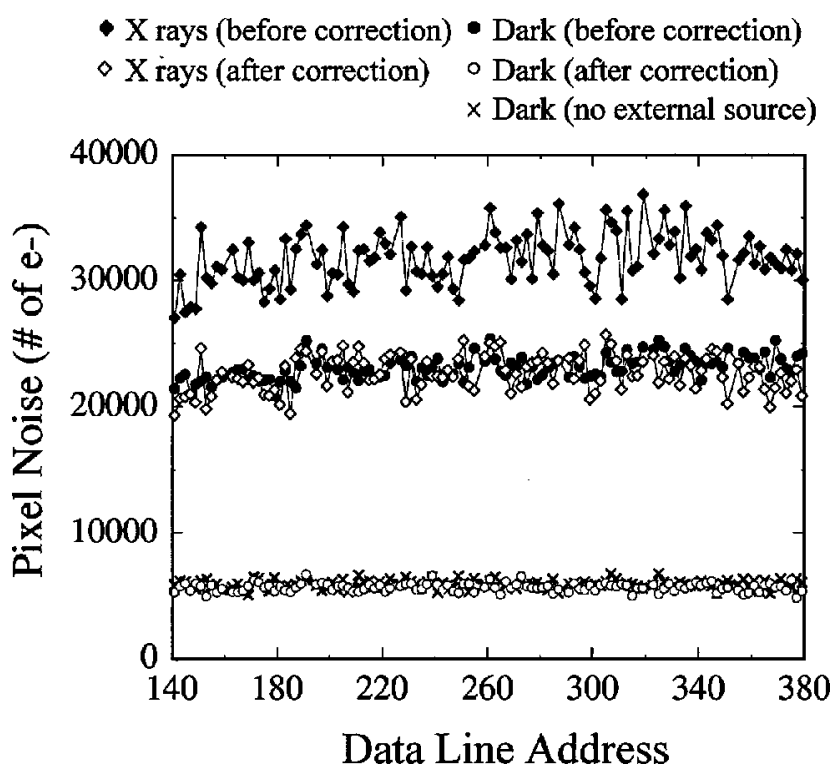

FIG. 4. Noise from individual pixels located along a single Gate line as a function of their corresponding Data line address. Results, obtained in the presence of an additional noise source and with no radiation, are shown before and after application of the compensation line correction (solid and open circles, respectively). For comparison, additional dark field results obtained in the absence of the external source are also shown (crosses). Results obtained with $\mathrm{x}$ rays are also shown before and after application of the compensation correction (solid and open diamonds, respectively). In all cases, the compensation correction was based on Eq. (1) using 14 pixels on each side of the region containing the imaging pixels.

\section{NPS measurements}

Noise power spectra were determined from flood field and dark field images such as those shown in Fig. 5. In this figure, sample images obtained in the absence of radiation [Figs. 5(a) and 5(b)] and with $x$ rays [Figs. 5(c) and 5(d)] are shown. The image data shown has had offset and gain corrections applied as described in Sec. II. These results were obtained both with [Figs. 5(b) and 5(d)] and without [Figs. $5(\mathrm{a})$ and $5(\mathrm{c})$ ] the use of the compensation line correction given by Eq. (1). The application of the compensation correction largely eliminates the strong horizontal correlations that are particularly evident in the dark field image [Fig. 5(a)]. Even after this correction, residual noise correlation effects are still present in Fig. 5(b), indicating that the correction technique is not completely effective.

The residual correlated noise seen in Fig. 5(b) is also observed in the NPS curves derived from dark field data shown in Fig. 6(a). In this figure, NPS determined along the Data line direction in the absence of the compensation line correction (solid circles) exhibits a strong correlation at $\sim 0.27 \mathrm{~mm}^{-1}$ that is associated with the horizontal striations evident in Fig. 5(a). This frequency corresponds to a pattern of noise repeated every $\sim 7.3$ lines in an image which, in turn, corresponds to $60 \mathrm{~Hz}$ noise pickup (given that 7.3 lines at $2.25 \mathrm{~ms}$ readout time per line requires $\sim 16.6 \mathrm{~ms}$ ). After the compensation correction is applied (open circles), the magnitude of the resulting NPS is reduced and the strong correlation at $\sim 0.27 \mathrm{~mm}^{-1}$ is suppressed, although residual correlated noise remains. 


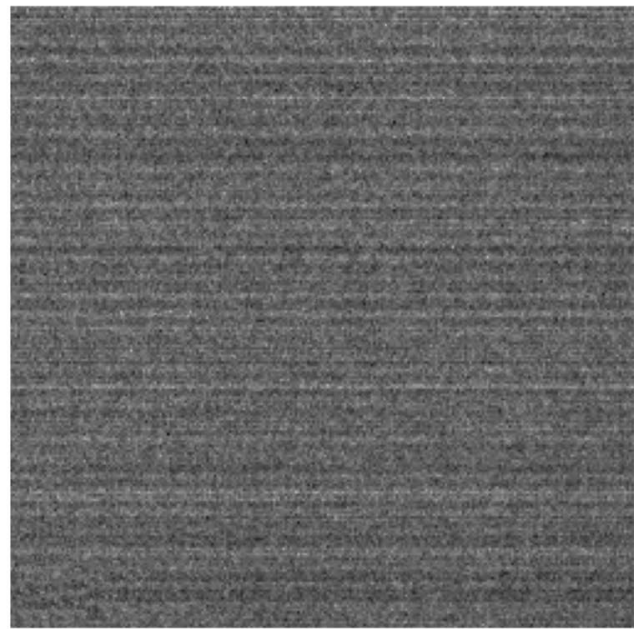

(a)

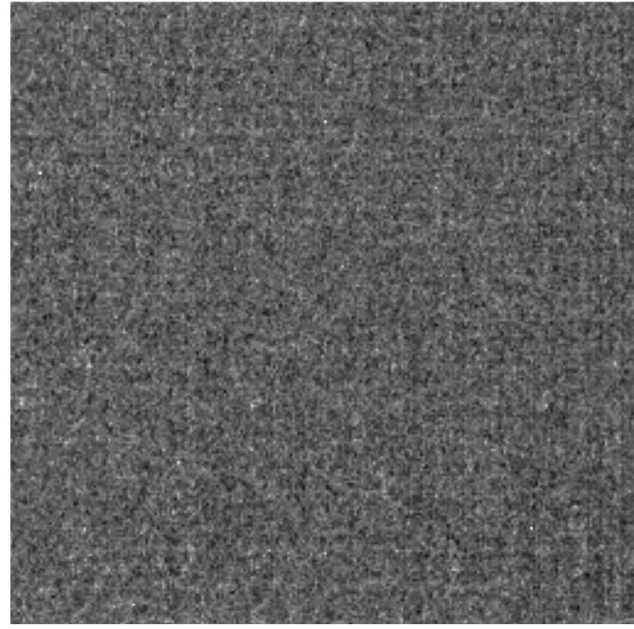

(c)

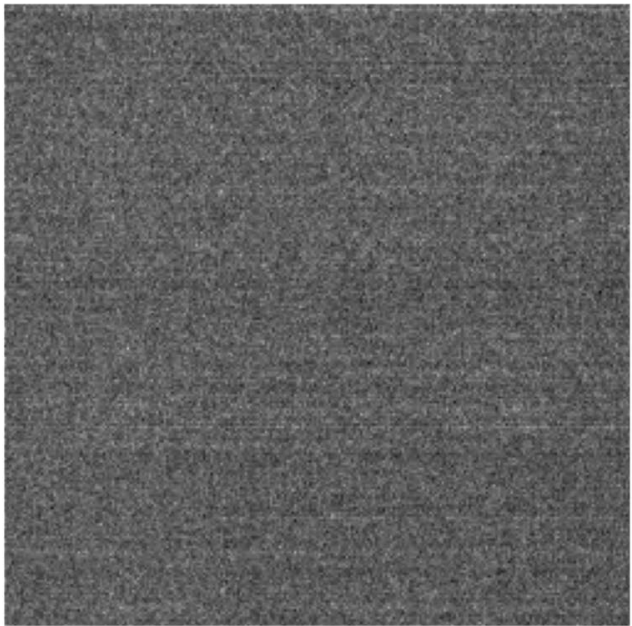

(b)

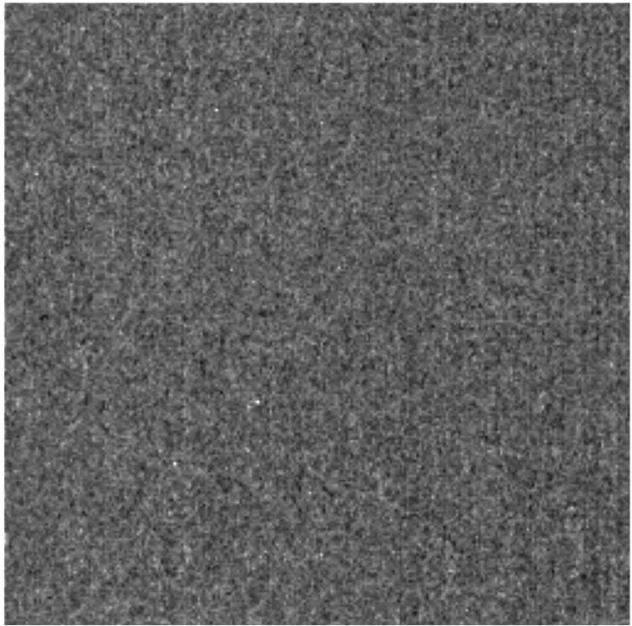

(d)

FIG. 5. Sample images with $256 \times 256$ pixels that were employed in deriving noise power spectra. The images were obtained in the absence of radiation [(a) and (b)] as well as with $\mathrm{x}$ rays [(c) and (d)]. The images in (b) and (d) include a compensation line correction while the images in (a) and (c) do not. The compensation correction was based on Eq. (1) using 14 compensation lines on each side of the region containing the imaging pixels. For each image, the gray scale window and level have been separately adjusted in order to maximize contrast.

To illustrate the efficiency of the correction, the NPS determined along the Gate line direction is also shown in Fig. 6 (a) (crosses). (By virtue of how the compensation line correction is defined, its application does not affect NPS along the Gate line direction and thus the Gate line NPS results remain unchanged whether the correction is applied or not.) Since pixels along the Gate line direction are not differentially affected by externally-coupled noise, the corresponding NPS is "white" and does not exhibit the strong correlations seen in the NPS determined along the Data line direction in the absence of the compensation correction. Conversely, even with the correction, the NPS determined along the Data line direction is higher in magnitude than the NPS corresponding to the Gate line direction. This indicates that the two directions are not equivalent in terms of additive noise-a difference most likely arising from the manner in which such arrays are read out. A similar pattern of behavior is observed in the case of NPS x-ray results obtained along the Data line direction with (open circles) and without (solid circles) the compensation line correction, as illustrated in Fig. 6(b). Once again, the strong correlation at $\sim 0.27 \mathrm{~mm}^{-1}$ is largely suppressed after the application of the compensation line correction. However, NPS results obtained with the compensation correction along the Data lines (open circles) and along the Gate lines (crosses) are essentially indistinguishable due to the dominance of $\mathrm{x}$-ray quantum noise.

\section{Imaging example}

Figure 7 shows images of an anthropomorphic phantom obtained with (b) and without (a) the use of the compensation line correction based on Eq. (1) and using 14 compensation lines from each side of the array. Comparison of the 

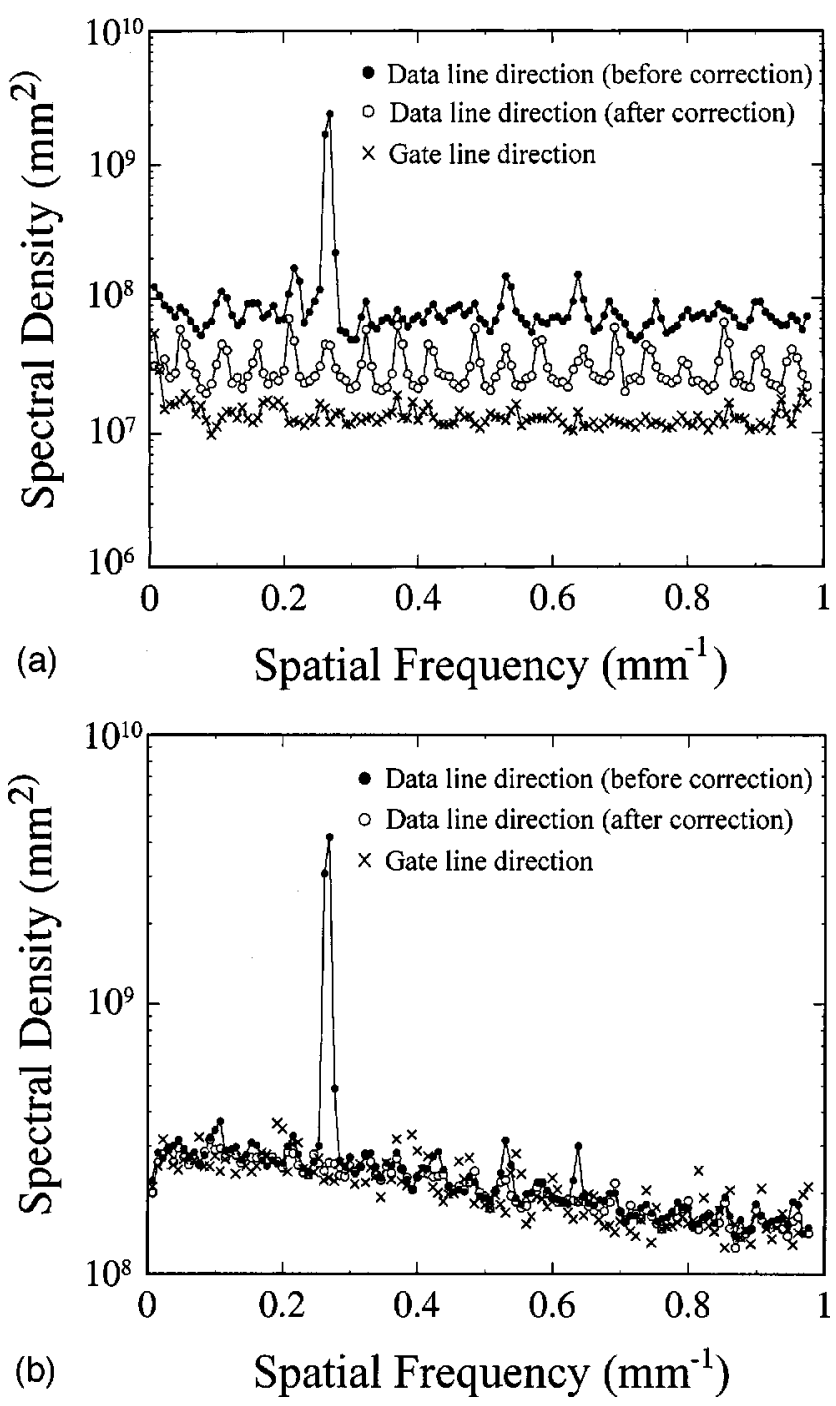

FIG. 6. One-dimensional noise power spectra (NPS) obtained (a) in the absence of radiation and (b) with $\mathrm{x}$ rays. Results obtained along the Data line direction are shown before and after application of the compensation line correction (solid and open circles, respectively). For comparison, results obtained in the orthogonal (Gate line) direction are also shown (crosses).

two images clearly demonstrates that the compensation correction removes most of the artifacts generated by the external noise source.

\section{DISCUSSION AND CONCLUSIONS}

A study of the use of compensation lines to reduce externally-coupled noise in indirect detection, active matrix flat-panel imagers has been reported. Compensation lines consist of columns of pixels, generally located along the periphery of an array, which are nonresponsive to radiation but which are as sensitive to external noise as normal imaging pixels. In this study, the effect of corrections, based on compensation line signals, on measurements of pixel signal, pixel noise, and noise power spectra was quantitatively examined. In addition, a qualitative demonstration of the use of compensation line corrections, involving images of a hand phantom, was presented.
In these studies, the effects of correlated external noise were examined both with an additional noise source (for the pixel signal, pixel noise, and image studies) and without the additional source (for the NPS studies). The use of this source served to more clearly demonstrate the effect of compensation line corrections as well as to illustrate that such corrections can effectively deal with relatively large external noise contributions. Moreover, in the absence of the added source, these contributions were non-negligible in comparison to x-ray quantum noise. Obviously, at exposures lower than those used in the present studies such as that encountered in fluoroscopy, these contributions would be relatively enhanced.

The results of these quantitative studies indicate that a correction based on signals derived from a single compensation line is highly effective in suppressing correlated noise originating from electronic noise sources external to the array. However, this form of the compensation line correction increases non-radiation-related uncorrelated noise. Fortunately, the introduction of multiple compensation lines, wherein the signal used for correction is derived from the average value from a number of compensation line pixels along a given row, reduces this effect. In addition, a positiondependent correction based on multiple compensation lines located on both sides of the array was found to be effective when the magnitude of the correlated noise varied linearly across the array. Moreover, the NPS studies demonstrate that this form of analysis is particularly effective in evaluating the degree to which compensation line corrections reduce external noise. For example, NPS obtained along the Data line direction in the absence of radiation exhibits a strong peak at $0.27 \mathrm{~mm}^{-1}$ corresponding to $60 \mathrm{~Hz}$ noise pickup. However, if a compensation correction is applied, the resulting NPS is reduced and the peak is largely suppressed. The resulting NPS, when compared to the NPS obtained along the orthogonal (Gate line) direction, exhibits a higher magnitude of additive noise. The difference in NPS values along the Gate and Data line is most likely a consequence of the row-by-row nature of array readout. Finally, while the compensation correction is effective in largely suppressing the correlated noise component of the external noise, it will not influence any contributions of the uncorrelated component.

In order for compensation line corrections to work properly, compensation line pixels should exhibit signal and noise characteristics identical to the behavior of normal, correctly functioning imaging pixels operated in the absence of radiation. Therefore, compensation pixels should exclude any defective pixels, should be insensitive to incident radiation, and should be in relatively close proximity to the imaging pixels. In the present study, the compensation pixels were, in fact, normal imaging pixels, which were shielded from incident light and radiation. Ideally, for an indirect detection AMFPI, compensation line pixels would be designed to be insensitive to radiation signal through the use of a reflector over the photodiodes. (Of course, there would still be some signal originating from direct interaction of the incident radiation within the photodiodes. ${ }^{5}$ ) For direct detection AMFPIs, the pixels would be designed so as to exclude 


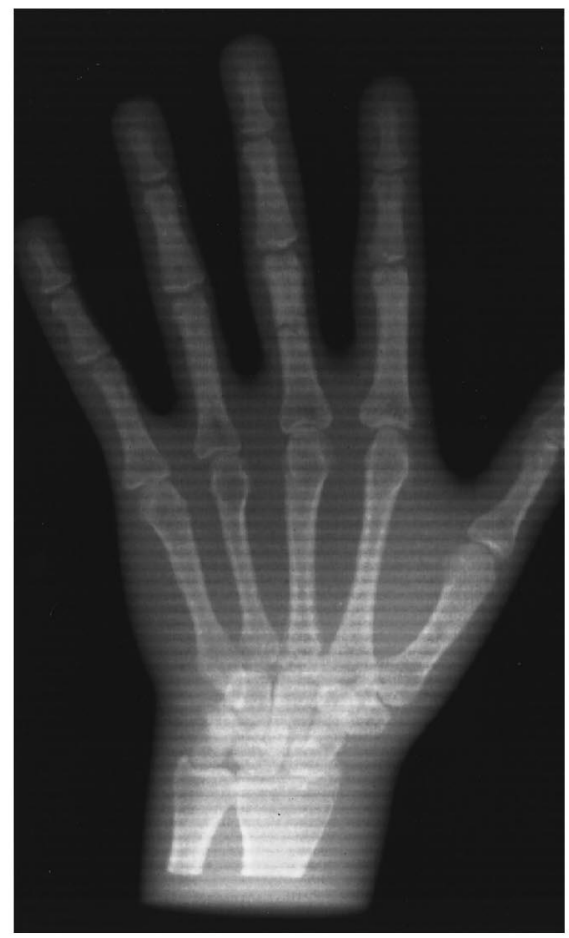

(a)

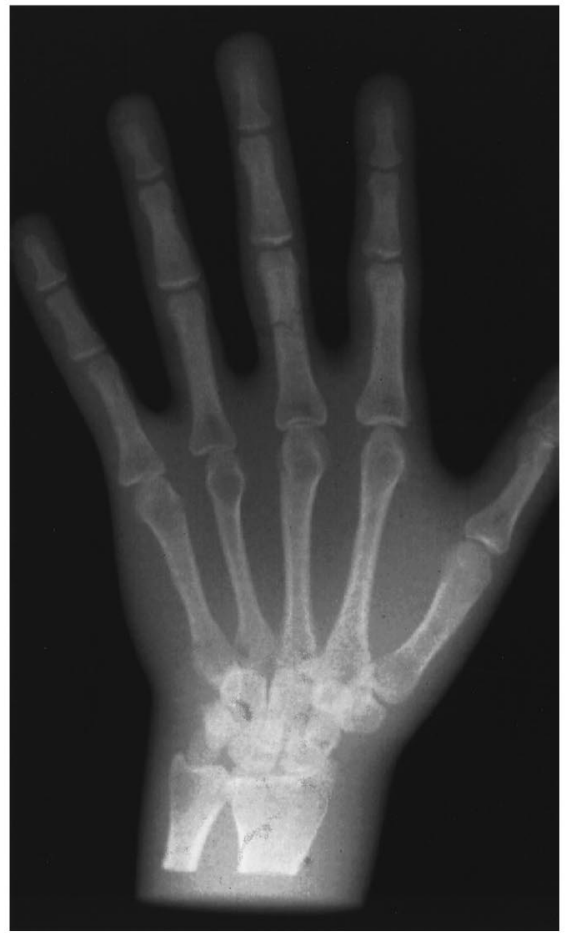

(b)

FIG. 7. Images of a hand phantom (a) without and (b) with the use of the compensation line correction based on Eq. (1). See text for further details.

collection of electron-hole pairs generated by the incident radiation in the overlying photoconductor. Alternatively, the array could be designed so as to have no photoconductive layer over the compensation lines-although this would alter the signal and noise characteristics of the corresponding pixels.

Close proximity of the compensation line pixels to the imaging pixels is important so as to insure that the external noise sampled by the compensation line pixels is representative of that sampled by the imaging pixels. Furthermore, the number and distribution of compensation lines in an array design should, ideally, be determined by the nature of the externally-coupled noise, $\sigma_{\text {ext }}$. In the cases that $\sigma_{\text {ext }}$ exhibits no variation, or a linear variation, along the length of the Gate lines, then a single group of compensation lines on one side of the array, or a pair of groups on either side of the array, will allow effective correction of correlated noise. However, if the externally-coupled noise varies in a nonlinear manner along the Gate lines, then a compensation line correction based on groups of compensation lines on both sides of the array may not provide an adequate correlated noise correction. In this case, a correction method based on a different distribution of compensation lines could be beneficial. For example, the inclusion of compensation lines regularly spaced throughout the array (e.g., every 100 lines) could largely circumvent the problem. Moreover, the gaps created in the resulting image can be readily corrected through the application of a median filter. ${ }^{22}$

It is worthwhile to point out that when a compensation line correction is to be performed, it should be done to the raw pixel data prior to any other image-processing step (such as offset and gain corrections or pixel defect filtration). Similarly, the compensation line correction should be applied to all raw pixel signal data that contribute to the creation of other image processing corrections (e.g., to the dark and flood field data used to generate offset and gain calibration constants). The application of other corrections before the compensation correction can result in an alteration of the correlated pixel-to-pixel signal variations along a Gate line, thereby rendering the compensation correction ineffective. Finally, although the compensation correction was performed off-line in this study, it can readily be performed immediately after image acquisition prior to real-time display of the image. ${ }^{26}$

\section{ACKNOWLEDGMENTS}

We gratefully acknowledge the support of Grant Nos. RO1-CA56135 and RO1-CA76405 from the National Institutes of Health.

\footnotetext{
${ }^{a)}$ Electronic mail: elmohri@umich.edu

${ }^{b}$ Present address: Department of Diagnostic Radiology, UT M.D. Anderson Cancer Center, Houston, TX 77030.

${ }^{1}$ L. E. Antonuk, Y. El-Mohri, J. Yorkston, K. W. Jee, J. Siewerdsen, M. Maolinbay, V. E. Scarpine, and H. Sandler, "Initial performance evaluation of an indirect-detection, active-matrix flat-panel imager (AMFPI) prototype for megavoltage imaging,' Int. J. Radiat. Oncol., Biol., Phys. 42, 437-454 (1998).
} 
${ }^{2}$ P. Munro and D. C. Bouius, "X-ray quantum limited portal imaging using amorphous silicon flat-panel arrays," Med. Phys. 25, 689-702 (1998).

${ }^{3}$ T. Falco, H. Wang, and B. G. Fallone, "Preliminary study of a metal/ $a$-Si-based portal detector,' Med. Phys. 25, 814-823 (1998).

${ }^{4}$ Y. El-Mohri, L. E. Antonuk, K-W. Jee, K. Brock, J. Balter, K. Lam, D. L. McShan, and B. A. Fraass, "Initial performance evaluation of a clinical prototype active-matrix flat-panel imager for radiotherapy imaging," Med. Phys. 25, 1583 (1998) (abstract).

${ }^{5}$ Y. El-Mohri, L. E. Antonuk, J. Yorkston, K. W. Jee, M. Maolinbay, K. L. Lam, and J. H. Siewerdsen, "Relative dosimetry using active matrix flat-panel imager (AMFPI) technology," Med. Phys. 26, 1530-1541 (1999).

${ }^{6}$ L. E. Antonuk, Y. El-Mohri, J. H. Siewerdsen, J. Yorkston, W. Huang, V. E. Scarpine, and R. A. Street, "Empirical investigation of the signal performance of a high-resolution, indirect detection, active matrix flatpanel imager (AMFPI) for fluoroscopic and radiographic operation," Med. Phys. 24, 51-70 (1997).

${ }^{7}$ J. H. Siewerdsen, L. E. Antonuk, Y. El-Mohri, J. Yorkston, W. Huang, J. M. Boudry, and I. A. Cunningham, "Empirical and theoretical investigation of the noise performance of indirect detection, active matrix flatpanel imagers (AMFPIs) for diagnostic radiology," Med. Phys. 24, 71-89 (1997)

${ }^{8}$ W. Zhao, I. Belvis, S. Germann, J. A. Rowlands, D. Waechter, and Z. Huang, "Digital radiology using active matrix readout of amorphous selenium: Construction and evaluation of a prototype real-time detector," Med. Phys. 24, 1834-1843 (1997).

${ }^{9}$ R. E. Colbeth et al., "Characterization of a third generation, multi-mode sensor panel," Proc. SPIE 3659, 491-500 (1999).

${ }^{10}$ P. R. Granfors, "Performance characteristics of an amorphous silicon flat panel x-ray imaging detector," Proc. SPIE 3659, 480-490 (1999).

${ }^{11}$ B. Polischuk et al., "Direct conversion detector for digital mammography," Proc. SPIE 3659, 417-425 (1999).

${ }^{12}$ S. Vedantham, A. Karellas, S. Suryanarayanan, D. Albagli, S. Han, E. J. Tkaczyk, C. E. Landberg, B. Opsahl-Ong, P. R. Granfors, I. Levis, C. J. D'Orsi, and R. E. Hendrick, "Full breast digital mammography with an amorphous silicon-based flat-panel detector: Physical characteristics of a clinical prototype," Med. Phys. 27, 558-567 (2000).

${ }^{13}$ M. Maolinbay, Y. El-Mohri, L. E. Antonuk, K.-W. Jee, S. Nassif, X. Rong, and Q. Zhao, "Additive noise properties of active matrix flat-panel imagers," accepted for publication in Med. Phys.

${ }^{14}$ L. E. Antonuk, K.-W. Jee, Y. El-Mohri, M. Maolinbay, S. Nassif, X. Rong, Q. Zhao, and J. H. Siewerdsen, "Strategies to improve the signal and noise performance of active matrix, flat-panel imagers for diagnostic x-ray applications," Med. Phys. 27, 289-306 (2000).
${ }^{15}$ R. A. Street, "Large area image sensor arrays," from Technology and applications of amorphous silicon, Springer series in Material Science, edited by R. A. Street (Springer, New York, 2000).

${ }^{16}$ R. E. Colbeth, M. J. Allen, D. J. Day, D. L. Gilblom, R. Harris, I. D. Job, M. E. Klausmeier-Brown, J. Pavlovich, E. J. Seppi, E. G. Shapiro, M. D. Wright, and J. M. Yu, "Flat-panel imaging system for fluoroscopy applications," Proc. SPIE 3336, 376-387 (1998).

${ }^{17}$ R. J. Yarema, T. Zimmermann, J. Srage, L. E. Antonuk, J. Berry, W. Huang, and M. Maolinbay, "A programmable, low noise, multichannel preamplifier for pixelated amorphous silicon arrays," Nucl. Instrum. Methods Phys. Res. A 439, 413-417 (2000).

${ }^{18}$ U. Schiebel, N. Conrads, N. Jung, M. Weibrecht, H. Wieczorek, T. Zaengel, M. J. Powell, I. D. French, and C. Glasse, "Fluoroscopic x-ray imaging with amorphous silicon thin-film arrays," Proc. SPIE 2163, 129-140 (1994).

${ }^{19}$ L. E. Antonuk, Y. El-Mohri, K.-W. Jee, M. Maolinbay, S. C. Nassif, X. Rong, J. H. Siewerdsen, Q. Zhao, and R. Street, "Beyond the limits of present active matrix, flat-panel imagers (AMFPIs) for diagnostic radiology," Proc. SPIE 3659, 518-527 (1999).

${ }^{20}$ R. L. Weisfield, M. A. Hartney, R. A. Street, and R. B. Apte, "New amorphous-silicon image sensor for $\mathrm{x}$-ray diagnostic medical imaging applications," Proc. SPIE 3336, 444-452 (1998).

${ }^{21}$ E. J. Morton, L. E. Antonuk, J. E. Berry, W. Huang, P. Mody, and J. Yorkston, "A data acquisition system for flat-panel imaging arrays," IEEE Trans. Nucl. Sci. 41, 1150-1154 (1994).

${ }^{22}$ L. E. Antonuk, J. Boudry, W. Huang, D. L. McShan, E. J. Morton, J. Yorkston, M. J. Longo, and R. A. Street, " Demonstration of megavoltage and diagnostic $\mathrm{x}$-ray imaging with hydrogenated amorphous silicon arrays," Med. Phys. 19, 1455-1466 (1992).

${ }^{23}$ J. C. Dainty, and R. Shaw, Image Science: Principles, Analysis and Evaluation of Photographic Type Imaging Processes (Academic, London, 1974).

${ }^{24}$ M. L. Giger, K. Doi, and C. E. Metz, "Investigation of basic imaging properties in digital radiography. 2. Noise Wiener spectrum,'” Med. Phys. 11, 797-805 (1984).

${ }^{25}$ A. D. A. Maidment and M. Yaffe, "Analysis of the spatial frequencydependent DQE of optically coupled digital mammography detectors," Med. Phys. 21, 721-729 (1994).

${ }^{26}$ W. Huang, L. E. Antonuk, J. Berry, M. Maolinbay, C. Martelli, P. Mody, S. Nassif, and M. Yeakey, "An asynchronous, pipelined, electronic acquisition system for active matrix flat-panel imagers (AMFPIs)," Nucl. Instrum. Methods Phys. Res. A 431, 273-284 (1999). 